PROCEEDINGS OF THE

AMERICAN MATHEMATICAL SOCIETY

Volume 133, Number 2, Pages 343-348

S 0002-9939(04)07692-0

Article electronically published on August 25, 2004

\title{
VALUATIONS AND RANK OF ORDERED ABELIAN GROUPS
}

\author{
MANISH KUMAR
}

(Communicated by Bernd Ulrich)

\begin{abstract}
It is shown that there exists an ordered abelian group that has no smallest positive element and that has no sequence of nonzero elements converging to zero. Some formulae for the rank of ordered abelian groups have been derived and a necessary condition for an order type to be rank of an ordered abelian group has been discussed. These facts have been translated to the spectrum of a valuation ring using some well-known results in valuation theory.
\end{abstract}

\section{INTRODUCTION}

An ordered abelian group, $(G,+)$, is a linearly ordered, abelian group such that the ordering is compatible with the group operation, i.e., if $x \geq 0$ and $y \in G$, then $x+y \geq y$ and $x \geq 0 \Leftrightarrow 0 \geq-x$. A subgroup $H$ of $G$ is an isolated subgroup if $H$ is a segment, i.e., if $x \in H$, then the interval $[-x, x] \subset H$. Let $k$ be a field. A homomorphism, $v$, from $\left(k^{*},.\right) \rightarrow G$ is a valuation if $v(x+y) \geq \min (v(x), v(y))$. $R_{v}=\{x \in k: v(x) \geq 0\} \cup\{0\}$ is the valuation ring and the image of $v$, denoted by $G_{v}$, is a subgroup of $G$ and is known as the value group of the valuation $v$. Ordered abelian groups play an imporant role in the study of valuation rings. These rings are of interest to algebraic geometers. Ideals of a valuation ring have close association with the isolated subgroups of the corresponding value group. In the next section, some well-known results in this direction are stated, which shall be used subsequently. A fact from topology has also been proved. In Section 3 an ordered abelian group has been constructed with no smallest positive element and which has no sequence of nonzero elements converging to zero. This with slight generalization allows us to construct, corresponding to any infinite cardinal $\lambda$, a valuation ring whose maximal ideal cannot be generated by any set of cardinality less than or equal to $\lambda$. Section 4 provides some formulae for the rank of an ordered abelian group which also enables us to explicitly construct groups with certain preassigned rank. A necessary condition for an order type to be the rank of an ordered abelian group has also been observed. The interested reader may also look up the appendix of $\mathrm{Ab} 2$ for results concerning embedding of an ordered abelian group of rank $S$ in $\mathbb{R}^{S}$, with lexicographic ordering.

Received by the editors April 25, 2003

2000 Mathematics Subject Classification. Primary 12F10, 14H30, 20D06, 20 E22.

Key words and phrases. Ordered abelian groups, spectrum of valuation rings.

The author thanks Prof. Shreeram S. Abhyankar for the motivation and support provided in developing the theory and in verifying the proof.

(C)2004 American Mathematical Society Reverts to public domain 28 years from publication 


\section{Preliminaries}

Result 1. There is a one-to-one inclusion reversing correspondence between the prime ideals of a valuation ring $R$ and the isolated subgroups of the corresponding value group $G$.

Proof. Let us denote the set of all isolated subgroups of $G$ by $\mathbb{F}(G)$ and the set of all prime ideals in $R$ by $\operatorname{spec}(R)$. The maps

$$
\phi: H \mapsto\{x \in R: v(x) \notin H\}
$$

and

$$
\psi: I \mapsto\{g \in G:|g|<v(x), \forall x \in I\}=\{v(y),-v(y): \forall y \in R \backslash I\}
$$

are defined on the set of all segments of $G$ and the set of all ideals in $R$ respectively. Clearly, $\phi(H)$ is an ideal and $\psi(I)$ is a segment. Also note that $\phi$ and $\psi$ are inverses of each other. These maps, when restricted to $\mathbb{F}(G)$ and $\operatorname{spec}(R)$ respectively, give the required bijection. For, if $H$ is also a subgroup, then $x, y \notin \phi(H) \Rightarrow v(x), v(y) \in$ $H \Rightarrow v(x y)=v(x)+v(y) \in H \Rightarrow x y \notin \phi(H)$. Hence $\phi(H)$ is prime. On the other hand, if $I$ is prime, then for $g, h \in \psi(I)$, let $x, y$ be such that $v(x)=g, v(y)=h$. Clearly $x, y \notin I$, which implies $x y \notin I \Rightarrow v(x)+v(y)=v(x y) \in \psi(I)$. Hence $\psi(I)$ is a subgroup.

We also know that if the value group is $\mathbb{Z}$, then all ideals are principal and if the value group is prediscrete, i.e., it has a smallest positive element, then the maximal ideal of the ring is principal. In fact, a more general result is true.

Result 2. With the notation of the previous result, let $H$ be a nonempty segment of $G$. A subset $A$ of $\phi(H)$ generates $\phi(H)$ if and only if whenever $g>0$ and $g<$ $\inf \{v(x): x \in A\}$, then $g \in H$.

Proof. For the "if part", let $z \in \phi(H)$, i.e., $v(z) \notin H$. $H$ being nonempty, $0 \in H$. So $v(z)>0$ and hence by hypothesis $v(z) \geq \inf \{v(x): x \in A\}$. This means that there exists $y \in A$ with $v(y) \leq v(z)$, so that $z \in y R_{v} \subset A R_{v}$. Hence $\phi(H)=A R_{v}$. "Only if part": Let $g \in G, 0<g<\inf \{v(x): x \in A\}$ and $y \in R_{v}$ be such that $v(y)=g$. Clearly, $y \notin A R_{v}=\phi(H)$; hence $g \in H$.

For any element $x$ in a well-ordered set $(T, \leq), I(x, T)=\{y \in T: y \leq x\}$ is called the segment of $x$ in $T$. It is known that there exists an uncountable well-ordered set $S$ every segment of which is countable. To see this, let $T$ be an uncountable well-ordered set, and let $A=\{x \in T: I(x, T)$ is uncountable $\}$. If $A=\emptyset$, then take $S$ to be $T$. If $A \neq \emptyset$, then, $A$ being well-ordered has a smallest element, say $a$. Let $S=I(a, T) \backslash\{a\}$. Then $S$ is an uncountable well-ordered set such that $I(b, S)$ is countable for every $b \in S$. Note that $S$ has no maximal element.

Remark 1 . We generalize the observations made in the previous paragraph. Let $T_{\lambda}$ be a well-ordered set of cardinality strictly greater than a given infinite cardinal $\lambda$. Let again $A=\left\{x \in T_{\lambda}:\left|I\left(x, T_{\lambda}\right)\right|>\lambda\right\}$. If $A \neq \emptyset$, then define $S_{\lambda}=I\left(a, T_{\lambda}\right) \backslash\{a\}$, where $a$ is the minimal element of $A$. Otherwise $S_{\lambda}=T_{\lambda}$. Hence we have a set of cardinality strictly greater than $\lambda$, every segment of which has cardinality less than or equal to $\lambda$. 


\section{Construction of A nON-PREDiscrete Detachable Group}

Definition 1. $H$ is real discrete if $H=\mathbb{Z}$, discrete if $H=\mathbb{Z}^{[d]}$ for some $d \in \mathbb{N}$, prediscrete if either $H=0$ or $H$ has a smallest positive element. $H$ is detachable if $H$ has no sequence of nonzero elements converging to zero, where a sequence $\left\{a_{n}\right\}$ is said to converge to zero if given any $\epsilon>0, \epsilon \in G$, there exist an integer $N \geq 1$ such that for all $n>N,\left|a_{n}\right|<\epsilon$.

It follows from Result 2 that $G$ has a sequence of nonzero elements converging to zero if and only if the maximal ideal of the corresponding valuation ring is countably generated. So if the value group is detachable and not prediscrete, then the maximal ideal of the corresponding valuation ring is not countably generated. The construction of an ordered abelian group with this property is given in the next paragraph. We know that given any ordered abelian group $G$, the set $k(X)_{G}$ of all maps from $G$ to $k$ with well-ordered support is naturally a field. Here $k$ is any field. On $k(X)_{G}$ define a valuation $v$, given by $v(f)=\min \operatorname{supp}(f)$. The value group for this valuation is clearly $G$. Hence this along with the group constructed below exhibits the existence of a valuation ring whose maximal ideal is not countably generated. In fact, Remark 2 generalizes this result to any cardinal.

Let $H$ be a nonzero ordered abelian group and $S$ be an uncountable well-ordered set, all of whose segments are countable. Let $G=H^{S}$ and make $G$ into an additive abelian group by componentwise addition. Declare $f<g$ to mean that upon letting $c$ be the smallest element in $S$ with $f(c) \neq g(c)$ we have $f(c)<g(c)$. Clearly $G$ has no smallest positive element, since $S$ has no maximal element. Let $\left(f_{n}\right)_{1 \leq n \leq \infty}$ be any sequence of nonzero elements in $G$ and for each $n$ let $c_{n}$ be the smallest element of $S$ with $f_{n}\left(c_{n}\right) \neq 0$. Now $\bigcup_{1<n<\infty} I\left(c_{n}, S\right)$ is countable since $I\left(c_{n}, S\right)$ is; hence there exists $d \in S$ with $d>c_{n}$ for all $n$. So $\left|f_{n}\right|>\epsilon$ where $\epsilon>0$ in $G$ is obtained by taking $\epsilon(a)=0$ for all $a<d$ in $S$ and $\epsilon(d)>0$ in $H$. Thus $G$ has no sequence of nonzero elements converging to zero.

Remark 2. In the above if we take $G=H^{S_{\lambda}}$, where $S_{\lambda}$ is as in Remark 1 , then the maximal ideal of the valuation ring with this value group is not generated by any set of cardinality less than or equal to $\lambda$. For, let $A$ be a subset of the maximal ideal with $|A| \leq \lambda$. Then $|\{v(x): x \in A\}| \leq \lambda$. By an argument similar to that in the above paragraph and using the fact that, for a set $I$, with $|I| \leq \lambda$ and sets $A_{i}, i \in I,\left|A_{i}\right| \leq \lambda$, where $\lambda$ is any infinite cardinal, $\left|\bigcup_{i \in I} A_{i}\right| \leq \lambda$, it follows that there exist $g \in G$ with $0<g<\inf \{v(x): x \in A\}$. So by Result 2, the assertion follows.

\section{ORDER type of RANK of ORDERED ABELIAN GROUPS}

Definition 2. Let $H$ be an ordered abelian group. We define the rank of $H$, $\rho(H)$, to be the linearly ordered set of all nontrivial isolated subgroups of $H$, with inclusion as the ordering.

Notation 1. If $S$ is a linearly ordered set, then $S \cup\{\infty\}$ denotes the set with an element $\infty$ augmented to it that is greater than every other element, and similarly $\{-\infty\} \cup S$ denotes the set with an element $-\infty$ augmented to it that is smaller than every other element.

Notation 2. If $S$ is a linearly ordered set, then $S^{\prime}$ denotes the same set with the reverse ordering. 
Definition 3. Let $I$ be a linearly ordered set and for each $i \in I$, let $S_{i}$ be a linearly ordered set. Define the concatenation of $S_{i}$ 's to be the set $\left\{(i, s): i \in I\right.$ and $\left.s \in S_{i}\right\}$, denoted by $\star_{i \in I} S_{i}$, with the ordering $(i, s) \leq(j, t)$ if $i<j$ or $i=j$ and $s \leq t$.

Being consistent with Notation $2, \star_{i \in I^{\prime}} S_{i}$ is $\left\{(i, s): i \in I\right.$ and $\left.s \in S_{i}\right\}$ with the ordering $(i, s) \leq(j, t)$ if $j<i$ or $i=j$ and $s \leq t$. Note that for $i, j \in I$, by $i<j$ we will always mean $i<_{I} j$ (and NOT $i<_{I^{\prime}} j$ ). Also observe that $\rho(G)=\rho\left(G^{\prime}\right.$ ).

Theorem 1. If I is well-ordered, for each $i \in I, \rho\left(G_{i}\right)=S_{i}$ and $G=\bigoplus_{i \in I} G_{i}$ with the ordering, $f<g$ if $f(c)<_{G_{c}} g(c)$ where $c=\min \{i: f(i) \neq g(i)\}$, then $\rho(G)=\star_{i \in I^{\prime}} S_{i}$.

Proof. Let $H \in S_{i}$, and define $\hat{H}=\{f: f(i) \in H$ and $f(j)=0$ for all $j<i\}$. Clearly, $\hat{H}$ is a subgroup of $G$. We shall show that it is an isolated subgroup, i.e., if $g \in G$ and $h \in \hat{H}$ with $|g|<_{G}|h|$, then $g \in H$. $|g|<_{G}|h|$ exactly means $|g(c)|<_{G_{c}}|h(c)|$ where $c=\min \{i: g(i) \neq h(i)\}$. So $h(c) \neq 0$ and hence, by definition of $\hat{H}, i \leq c$.

So $g(j)=h(j)=0 \forall j<i$ and $|g(i)| \leq_{G_{i}}|h(i)| ;$ in particular, $g(i) \in H, H$ being isolated. Hence $g \in \hat{H}$. This enables us to define the map $(i, H) \mapsto \hat{H}$ from $\star_{i \in I^{\prime}} S_{i} \rightarrow \rho(G)$. Clearly this map is injective.

We shall first show that it is surjective and later that it is order preserving. This would complete the proof.

Let $T$ be a nontrivial isolated subgroup of $G$. We need to show that $T=\hat{H}$ for some $H \in S_{i}$ for some $i \in I$. Let $J=\left\{i: T \supset \bigoplus_{j>i} G_{j}\right\}$. Let $f \neq 0$ belong to $T$ and $k$ be such that $f(k) \neq 0$. If $g \in \bigoplus_{j>k} G_{j}$, then $|g|<_{G}|f| \Rightarrow g \in T$; hence $k \in J$. Therefore, $J$ is a nonempty subset of a well-ordered set. Let $c=\min (J)$.

Now, if $f \in T$ and $d=\min \operatorname{supp}(f)$, then $d \geq c$ and hence $f \in \bigoplus_{j \geq d} G_{j} \subset$ $\bigoplus_{j \geq c} G_{j}$. So we have

$$
\bigoplus_{j \geq c} G_{j} \supset T \supset \bigoplus_{j>c} G_{j}
$$

Let $H=\{f(c): f \in T\} . H$ is trivially an isolated subgroup of $G_{c}$. Finally, we claim that $T=\hat{H}$.

$f \in T \Rightarrow f(c) \in H$ and $f(i)=0$ for all $i<c$, since $\bigoplus_{j \geq c} G_{j} \supset T$. Hence $f \in \hat{H}$.

Also, $f \in \hat{H} \Rightarrow \exists g \in T$ such that $f(c)=g(c) \Rightarrow f-g \in \bigoplus_{j>c} G_{j} \subset T \Rightarrow f \in T$.

It remains to see that the map is order preserving. Let $(i, H) \leq(j, K)$. If $i=j$, then $H \subset K$; hence $\hat{H} \subset \hat{K}$ and if $i>j$, then $\hat{K} \supset \bigoplus_{k>j} G_{k} \supset \bigoplus_{k \geq i} G_{k} \supset \hat{H}$.

Theorem 2. If $I$ is well-ordered, for each $i \in I, \rho\left(G_{i}\right)=S_{i}$ and $G=\bigoplus_{i \in I} G_{i}$ with the ordering, $f<g$ if $f(c)<_{G_{c}} g(c)$ where $c=\max \{i: f(i) \neq g(i)\}$, then $\rho(G)=\star_{i \in I} S_{i}$ if $I$ has a maximal element, and otherwise $\rho(G)=\star_{i \in I} S_{i} \cup\{\infty\}$.

Proof. Let $H \in S_{i}$, define $\hat{H}=\{f: f(i) \in H$ and $f(j)=0$ for $j>i\}$. That $\hat{H}$ is an isolated subgroup of $G$ follows similarly as in the previous proof. It allows us to define the map $(i, H) \mapsto \hat{H}$ from $\star_{i \in I} S_{i} \rightarrow \rho(G)$ and again this map is clearly injective.

For surjectivity, let $T$ be a nontrivial isolated subgroup of $G$ and $J=\{i: T \subset$ $\left.\bigoplus_{j \leq i} G_{j}\right\}$. If $J=\emptyset$, then $T \supset \bigoplus_{j \leq i} G_{j}$ for all $i \in I \Rightarrow T=G$. Now, if $m=\max (I)$ exists, then $T=\hat{G}_{m}=G$; otherwise $\infty \mapsto T$. When $J \neq \emptyset$, let $c=\min (J)$. We 
shall show

$$
\bigoplus_{j \leq c} G_{j} \supset T \supset \bigoplus_{j<c} G_{j} .
$$

The first inclusion is obvious. Let $f \in \bigoplus_{j<c} G_{j}$ and $d=\max \operatorname{supp}(f)$. Then $d<c \Rightarrow f \in \bigoplus_{j \leq d} G_{j} \subset T$, since $\bigoplus_{j \leq d} G_{j}$ is an isolated subgroup of $G$ and $T \not \subset \bigoplus_{j \leq d} G_{j}$. Hence the second inclusion.

Let $H=\{f(c): f \in T\} . H$ is again an isolated subgroup of $G_{c}$ and, as in previous proof, $T=\hat{H}$.

Finally, we need to see that the map is order preserving. Let $(i, H) \leq(j, K)$. If $i=j$, then $H \subset K$; hence $\hat{H} \subset \hat{K}$ and if $i<j$, then $\hat{K} \supset \bigoplus_{k<j} G_{k} \supset \bigoplus_{k \leq i} G_{k} \supset$ $\hat{H}$.

Now, we move on to show one interesting corollary of the two theorems above.

Corollary. There exist ordered abelian groups with rank $\mathbb{N} \cup\{\infty\}, \mathbb{Z} \cup\{\infty\}$ and $\mathbb{N}^{\prime}$. Hence there exist valuation rings with $\mathbb{N} \cup\{\infty\},\{-\infty\} \cup \mathbb{Z} \cup\{\infty\}$ and $\{-\infty\} \cup \mathbb{N}^{\prime}$ as the order type of its prime spectrum.

Proof. $\rho(\mathbb{Z})$ is clearly a singleton set, the whole group being the only nontrivial isolated subgroup. Hence with $I=\mathbb{N}, G_{i}=\mathbb{Z} \forall i$, applying Theorem 1 and Theorem 2 , we get ordered abelian groups, say, $K_{1}$ and $K_{2}$, with $\rho\left(K_{1}\right)=\mathbb{N}^{\prime}$ and $\rho\left(K_{2}\right)=$ $\mathbb{N} \cup\{\infty\}$ respectively. Now, applying Theorem 2 with $I=\{1,2\}$ to $K_{1}$ and $K_{2}$, we get another ordered abelian group, say $K$, with $\rho(K)=\mathbb{Z} \cup\{\infty\}$.

Recall that for an ordered abelian group $G, \rho(G)$ differs from $\mathbb{F}(G)$, the set of all isolated subgroups, by the trivial subgroup only. By Result 1 , we know that $\mathbb{F}(G)^{\prime}$ is order isomorphic to $\operatorname{spec}\left(R_{G}\right)$, where $R_{G}$ is any valuation ring with the value group $G$.

So we get that $\operatorname{spec}\left(R_{K_{1}}\right)$ is order isomorphic to $\mathbb{N} \cup\{\infty\}, \operatorname{spec}\left(R_{K_{2}}\right)$ is order isomorphic to $\{-\infty\} \cup \mathbb{N}^{\prime}$ and $\operatorname{spec}\left(R_{K}\right)$ is order isomorphic to $\{-\infty\} \cup \mathbb{Z} \cup\{\infty\}$.

Finally, we give a necessary condition for a linearly ordered set to be the rank of an ordered abelian group $G$. Note that if $A \subset \rho(G)$, then $K=\bigcup_{H \in A} H$ is an isolated subgroup. Also note that $K=\sup A$. Hence we have the condition that every subset of $\rho(G)$ has a supremum. Similarly every subset of $\rho(G)$ that is bounded below has an infimum. The intersection being nontrivial does the job. Some trivial consequences of this condition are that $\mathbb{Z}, \mathbb{N}, \mathbb{Q}$ or $\mathbb{Q} \cup\{\infty\}$ cannot be the rank of any ordered abelian group.

A necessary condition for a partially ordered set to be the prime spectrum of a ring, which could be found in [LO], when translated to ordered abelian groups says that if $H_{1}, H_{2} \in \rho(G)$ with $H_{1} \subsetneq H_{2}$, then there exist $K_{1}, K_{2} \in \rho(G)$ with $K_{2}$ the successor of $K_{1}$ and $H_{1} \subset K_{1} \subsetneq K_{2} \subset H_{2}$. This condition tells us that $\mathbb{R} \cup\{\infty\}$ cannot be the rank of an ordered abelian group.

Interestingly, the above two necessary conditions together turn out to be sufficient for a linearly ordered set to be $\rho(G)$ for some ordered abelian group $G$. This was observed by S. S. Abhyankar, Hagen Knaf and Franz-Viktor Kuhlmann recently and was communicated to me by Prof. Abhyankar.

\section{REFERENCES}

[Ab1] S. S. Abhyankar, Ramification Theoretic Methods in Algebraic Geometry, Princeton University Press, 1959. MR 0105416 (21:4158) 
[Ab2] S. S. Abhyankar, On valuations centered in the local domain, American Journal of Mathematics, 78 (1956), 321-348. MR 0082477 (18:556b)

[Mun] J. R. Munkres, A First Course in Topology, Prentice Hall Inc., 1975. MR $0464128(57: 4063)$

[LOh] W. J. Lewis, J. Ohm, The ordering of SpecR, Canadian Journal of Mathematics 28 (1976), no. 4, 820-835. MR 0409428 (53:13183)

Department of Mathematics, Purdue University, West Lafayette, Indiana 47907

E-mail address: mkumar@math.purdue.edu

$U R L:$ http://www . math.purdue.edu/ ${ }^{\sim}$ mkumar 